\title{
High energy neutrinos from charm in astrophysical sources
}

\author{
Rikard Enberg, ${ }^{1, *}$ Mary Hall Reno, ${ }^{2}$ and Ina Sarcevic ${ }^{1,3}$ \\ ${ }^{1}$ Department of Physics, University of Arizona, Tucson, AZ 85721 \\ ${ }^{2}$ Department of Physics and Astronomy, University of Iowa, Iowa City, IA \\ ${ }^{3}$ Department of Astronomy, University of Arizona, Tucson, AZ 85721
}

\begin{abstract}
Charm production gives rise to a flux of very high energy neutrinos from astrophysical sources with jets driven by central engines, such as gamma ray bursts or supernovae with jets. The neutrino flux from semi-leptonic decays of charmed mesons is subject to much less hadronic and radiative cooling than the conventional flux from pion and kaon decays and therefore has a dominant contribution at higher energies, of relevance to future ultrahigh energy neutrino experiments.
\end{abstract}

\section{INTRODUCTION}

Large underground or underwater experiments like IceCube [1] and KM3NeT 2] are designed with the goal of observing high energy neutrinos produced in astrophysical sources. The highest energy neutrinos, with energies of $10^{9} \mathrm{GeV}$ and higher may be observed in radio detection experiments 3], and with an even higher energy threshold of $10^{12} \mathrm{GeV}$ with acoustic detection experiments [4]. We consider astrophysical sources driven by a relativistic jet outflow, accelerated by a central engine such as a black hole [5, 6]. Shock accelerated protons in the jet outflow may give rise to a high-energy neutrino flux [7]. These neutrinos are potentially produced in hadronic interactions: proton-proton interactions produce charged pions and kaons which subsequently decay into muons and neutrinos. Above the threshold for $\Delta^{+}$production, proton interactions with ambient photons also produce charged pions, and at higher energies, kaons. The relative importance of the $p p$ and $p \gamma$ contributions to the neutrino fluxes depends on the characteristics of the astrophysical environment. Several types of astrophysical sources have been studied in e.g. 7, , 8, 9, 10, 11, 12, 13, 14, 15, 16, 17].

High energy pions and kaons are relatively long-lived and therefore subject to both hadronic and radiative cooling before they decay, which downgrades the neutrino energies. Charm production and decay in astrophysical jets is also a source of neutrinos [8]. In this paper, we show that production of charmed mesons in $p p$ collisions gives a large contribution to the neutrino flux at the highest energies, since high energy charmed hadrons $\left(D^{ \pm}, D^{0}\right)$ have short lifetimes and therefore predominantly decay before they interact. Moreover, since the amount of radiative cooling scales as $m^{-4}$, the larger masses of the charmed hadrons lead to less cooling. The neutrino flux from charm is therefore less suppressed up to higher energies. Even though the production cross section is orders of magnitude smaller than for pions and kaons, neutrinos from charm decays become the dominant contribution at high energies. The energy at which

\footnotetext{
${ }^{*}$ Present address: Department of Physics and Astronomy, Uppsala University, Sweden
}

charm begins to dominate depends on the detailed properties of the astrophysical source, and the extent to which charm contributes depends on the maximum energy of the accelerated protons in the jet.

An example of an astrophysical source model is the slow-jet supernova (SJS) model, proposed by Razzaque, Mészáros and Waxman (RMW) [9]. It is characterized by a mildly relativistic jet propagating in a collapsing star. This jet does not emerge from the source, and is sometimes referred to as a 'choked jet.' This environment has a large optical depth [10, 11], so neutrinos may be the only high energy signals to emerge. On the other hand, the jet in a Gamma Ray Burst (GRB) (see e.g. [6] for a review) is highly relativistic and the optical depth might be such that photons escape during the burst and do not thermalize. Other GRBs may be choked, so that only neutrinos escape [12].

We present here a new analysis of the neutrino flux for two types of source environments: the SJS and a GRB model, both of which have large magnetic fields in the jets. Our treatment of the proton-proton collisions accounts for charmed meson production, as well as pion and kaon production. The shorter lifetimes of charmed mesons allow this channel to potentially dominate the neutrino flux from $p p$ collisions.

\section{ASTROPHYSICAL SOURCES}

Cooling times for protons and mesons depend on the proton and photon densities in the jet, which are determined from the characteristics of the environment. The internal shock radius of the jet is given by $r_{j}=2 \Gamma_{j}^{2} c t_{v}$, where $\Gamma_{j}$ is the bulk Lorentz gamma factor of the jet outflow and $t_{v}$ is the variability time scale of the central engine. The proton and electron number densities can be calculated in terms of the jet luminosity, $L_{j}$, and the jet opening angle, $\theta_{j}$, as

$$
n_{e}^{\prime}=n_{p}^{\prime}=\frac{L_{j}}{2 \pi \theta_{j}^{2} r_{j}^{2} \Gamma_{j}^{2} m_{p} c^{3}} .
$$

The primes denote quantities in the frame comoving with the jet. The average magnetic field generated in the jet 
is given by

$$
B^{\prime}=\left(\frac{4 \epsilon_{B} L_{j}}{\theta_{j}^{2} r_{j}^{2} \Gamma_{j}^{2} c}\right)^{1 / 2}
$$

where $\epsilon_{B}$ is the fraction of kinetic jet energy converted into magnetic fields. The comoving energy density of photons in the jet is

$$
U_{\gamma}^{\prime}=\frac{\epsilon_{e} L_{j}}{2 \pi \theta_{j}^{2} r_{j}^{2} \Gamma_{j}^{2} c},
$$

where $\epsilon_{e}$ is the fraction of the energy radiated into photons.

Depending on the optical depth, in some astrophysical sources photons may be thermalized. For a thermal photon distribution with temperature $T^{\prime}$, the photon number density is given by $n_{\gamma}^{\prime} \propto\left(k T^{\prime}\right)^{3}$, leading to

$$
n_{\gamma}^{\prime}=\frac{2 \zeta(3)}{\pi^{7 / 2}}\left(\frac{15 U_{\gamma}^{\prime}}{\hbar c}\right)^{3 / 4}
$$

In the astrophysical environments where photons are not thermalized, the photon energies follow a broken powerlaw spectrum [18], characterized by the break energy $E_{\gamma, b}^{\prime}$, taken as a free parameter, and $U_{\gamma}^{\prime}=n_{\gamma}^{\prime} E_{\gamma}^{\prime}$.

\section{A. Slow Jet Supernova Model}

The RMW model of this system has a bulk jet Lorentz factor $\Gamma_{j}=3$. The characteristic time variability of the source is $t_{v}=0.1 \mathrm{~s}$, with a jet luminosity of $L_{j}=$ $3 \times 10^{50} \mathrm{erg} / \mathrm{s}$ and jet angle of $\theta_{j} \sim 1 / 3$. We take the energy fractions $\epsilon_{e, B}=0.1$. With these parameters, the estimated size of the jet is on the order of $10^{11} \mathrm{~cm}$, below a typical stellar radius for a pre-supernova star. Due to the large optical depth, photons thermalize.

\section{B. Gamma Ray Bursts}

We also consider a generic case of a GRB. There is a wide range of possible parameters and types of progenitors of GRBs, and the choice of parameters we make here is to be considered as just one example of a GRB-like jet. The jet gamma factor can vary over a large range of values, and the medium can be optically thick or thin. We choose the case of a GRB where the photons are not in thermal equilibrium and we take the values $\Gamma_{j}=100$, $L_{j}=3 \times 10^{50} \mathrm{erg} / \mathrm{s}, \theta_{j}=0.1, t_{v}=10^{-3} \mathrm{~s}$. We fix the photon break energy to be $E_{\gamma, b}^{\prime}=2.5 \mathrm{keV}$ [19] and the spectral indices to be -1 below and -2.25 above the break energy. We take the same energy fractions, $\epsilon_{e, B}$, as for the SJS model.

In Table I we show the number densities of protons and photons, the values of the magnetic field and the characteristic photon energy, in the comoving frame, for the SJS and GRB models.
TABLE I: The jet bulk Lorentz factor $\Gamma_{j}$, and the comoving number densities of protons $n_{p}^{\prime}$ and photons $n_{\gamma}^{\prime}$, average photon energy $E_{\gamma}^{\prime}$, and magnetic field in the jet $B^{\prime}$ for the slow-jet supernova (SJS) and gamma ray burst (GRB) models.

\begin{tabular}{lccccc}
\hline \hline Source & $\Gamma_{j}$ & $n_{p}^{\prime}\left[\mathrm{cm}^{-3}\right]$ & $B^{\prime}[\mathrm{G}]$ & $E_{\gamma}^{\prime}[\mathrm{keV}]$ & $n_{\gamma}^{\prime}\left[\mathrm{cm}^{-3}\right]$ \\
SJS & 3 & $3.6 \times 10^{20}$ & $1.2 \times 10^{9}$ & 4.5 & $2.8 \times 10^{24}$ \\
GRB & 100 & $3 \times 10^{16}$ & $1.1 \times 10^{7}$ & 2.5 & $1.1 \times 10^{21}$ \\
\hline
\end{tabular}

\section{TIME AND DISTANCE SCALES}

The neutrino flux is determined by the flux of accelerated protons, the production of mesons, meson interactions and meson decay. This all occurs in an environment of magnetic fields, photons, and a non-relativistic baryon density. To begin with, we consider the characteristic time scales and distance scales relevant to the accelerated protons. Since many of the processes are relevant to mesons as well, we also indicate the application to mesons.

Protons are accelerated by Fermi shock acceleration. The acceleration time is

$$
t_{\mathrm{acc}}^{\prime} \simeq 3 \times 10^{-12} \mathrm{~s} \frac{\kappa}{10} \frac{0.3}{\epsilon_{B}^{1 / 2}}\left(\frac{E_{p}^{\prime}}{\mathrm{GeV}}\right)\left(\frac{B^{\prime}}{10^{9} \mathrm{G}}\right)^{-1}
$$

where the the parameter $\kappa$ is inversely related to the diffusion coefficient. The details of the shock acceleration, in particular the orientation of the magnetic field relative to the shock, determine the value of diffusion coefficient 20]. Using the standard choice of $\epsilon_{B}=0.1$ and $\kappa=10$, the acceleration time converted to a distance is

$$
L_{N}^{\mathrm{acc}}=10^{-2} \mathrm{~cm}\left(\frac{E_{p}}{\mathrm{GeV}}\right)\left(\frac{B^{\prime}}{10^{9} \mathrm{G}}\right)^{-1} .
$$

A value of $\kappa=10$ taken here could be reduced by a factor of 10 or more depending on the orientation of the magnetic field [20, 21]. We comment below on the implications of a smaller value of $\kappa$.

Until proton cooling times (or proton interaction lengths) are smaller than the acceleration time, the proton energy spectrum within the jet is characterized by a power law which we take to be $\phi_{N} \sim\left(E_{p}^{\prime}\right)^{-2}$. We neglect shock acceleration of the charged mesons to simplify the evaluation [11].

The time scales for proton cooling depend on the comoving energies, particle densities, cross sections, and magnetic fields. Generically, the cooling time is $t_{\text {cool }}^{\prime}=E^{\prime} /\left|d E^{\prime} / d t^{\prime}\right|$. One can approximate $\left|d E^{\prime} / d t^{\prime}\right| \simeq$ $c n^{\prime} \sigma \Delta E^{\prime}$, where $\Delta E^{\prime}$ is the particle energy loss and $\sigma$ is the cross section of the hadron scattering with particles with comoving number density $n^{\prime}$. For scattering on photons, we use $\left\langle n^{\prime} \sigma v\right\rangle$, averaged over the photon spectrum.

There are several processes that contribute to the proton and/or meson cooling. The effect of the energy loss, accounting for the weighting by the incident flux, can be 
described by a cascade equation written in terms of $Z$ moments [22]. For the proton flux $\left(\phi_{N}\right)$ the propagation over distance $x^{\prime}$ in the co-moving jet frame is given by

$$
\left.\frac{d \phi_{N}}{d x^{\prime}}\right|_{\text {cool }}=-\frac{\phi_{N}}{\ell_{N}^{\text {had }}}+Z_{N N}^{\text {had }} \frac{\phi_{N}}{\ell_{N}^{\text {had }}}+\ldots
$$

where $\ell_{N}^{\text {had }}=\left(\sigma_{p p} n_{p}^{\prime}\right)^{-1}$ describes interactions with target protons. The ellipsis signifies other cooling mechanisms, such as $p \gamma$ reactions, and electromagnetic cooling, to be discussed below. The $Z$-moment is defined by

$$
Z_{N M}=\int_{0}^{1} d x_{E} x_{E}^{\alpha-1} \frac{d n_{N \rightarrow M}}{d x_{E}}
$$

where the flux of $N$ is $\phi_{N} \sim\left(E^{\prime}\right)^{-\alpha}, x_{E} \equiv E_{M}^{\prime} / E_{N}^{\prime}$ and $d n / d x_{E}$ describes the cross section normalized energy distribution of the meson $M$ produced by $N$ (or from $N$ decay).

Using the comoving proton number density from Table I and the cross section $\sigma_{p p} \simeq 5 \times 10^{-26} \mathrm{~cm}^{2}$, the interaction length is $\ell_{N}^{\text {had }} \simeq 5.5 \times 10^{4} \mathrm{~cm}$ for the SJS model of RMW, and $\ell_{N}^{\text {had }} \simeq 6.8 \times 10^{8} \mathrm{~cm}$ for the GRB. We used these same interaction lengths for the hadronic interaction of the mesons. Using the exact value for the inelastic cross section for mesons does not affect the result because the hadronic cooling time for mesons is much larger than its radiative cooling times.

The distance scale corresponding to the hadronic cooling time is

$$
L_{N}^{\mathrm{had}}=\frac{\ell_{N}^{\mathrm{had}}}{1-Z_{N N}^{\mathrm{had}}} \simeq c t_{N}^{\mathrm{had}}
$$

The $Z$-moment takes the inelasticity into account, and is equivalent to setting $\Delta E^{\prime} / E^{\prime} \simeq 1-Z_{N N}^{\text {had }}$ for this hadronic scattering process. We will use $Z_{N N}^{\text {had }}=0.5$ [23].

In addition to hadronic interactions, protons can lose energy via photon interactions such as inverse Compton (IC) scattering, where the proton loses energy to the ambient photons, or through inelastic $p \gamma$ interactions if the proton energy is above the $\Delta^{+}$threshold. For the IC process, as long as the center of mass energy is small compared to the proton mass, one can use the Thomson cross section, $\sigma_{T p}=\sigma_{T}\left(m_{e}^{2} / m_{p}^{2}\right)$. This approximation is good for protons scattering off $\mathrm{keV}$ photons for energies up to $E_{p}^{\prime} \sim 10^{5} \mathrm{GeV}$. Above this energy, IC becomes irrelevant. The effective scattering length for inverse Compton scattering is

$$
L_{N}^{I C}=\frac{3 m_{p}^{4} c^{4}}{4 \sigma_{T} m_{e}^{2} E_{p}^{\prime} U_{\gamma}^{\prime}}
$$

This effective scattering length is rescaled by $\left(m_{M} / m_{p}\right)^{4}$ for mesons.

For $E_{\gamma}^{\prime}=5 \mathrm{keV}$, the threshold energy for $\Delta^{+}$production in $p \gamma$ interactions is $E_{p, \text { th }}^{\prime}=2 \times 10^{5} \mathrm{GeV}$. For $p \gamma$ scattering, the averaged reaction rate is given by,

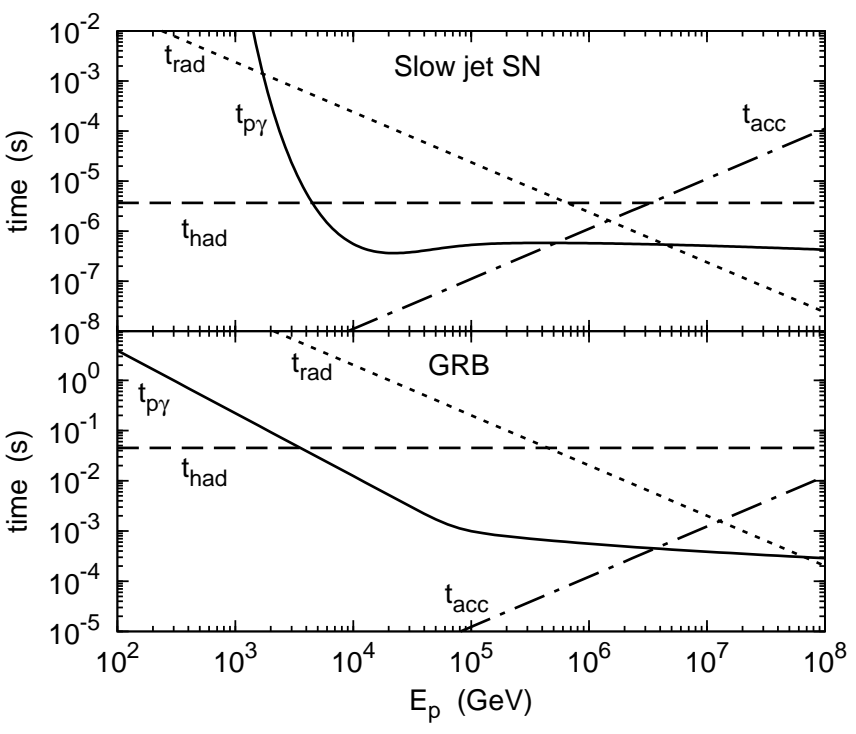

FIG. 1: Proton cooling times for SJS and for GRB in the frame co-moving with the jet.

$$
\left\langle n^{\prime} \sigma v\right\rangle=\frac{c}{8 \beta_{p}^{\prime} E_{p}^{\prime 2}} \int d E_{\gamma}^{\prime} \frac{\hat{n}_{\gamma}\left(E_{\gamma}^{\prime}\right)}{E_{\gamma}^{\prime 2}} \int d s\left(s-m_{p}^{2}\right) \sigma_{p \gamma}(s),
$$

where $\beta_{p}^{\prime}$ is the proton beta factor, $s$ is the center-of-mass energy squared, and $\hat{n}_{\gamma}\left(E_{\gamma}^{\prime}\right) d E_{\gamma}^{\prime}$ is the photon number density in the energy range between $E_{\gamma}^{\prime}$ and $E_{\gamma}^{\prime}+d E_{\gamma}^{\prime}$. The cross section $\sigma_{p \gamma}$ is given by the resonance plus continuum multiparticle production contributions given in Ref. [24]. The photon distribution $\hat{n}_{\gamma}$ is thermal for the SJS and a power law for the GRB [7]. In determining the cooling time, $t_{p \gamma}^{\prime}$, we take $\Delta E^{\prime} / E^{\prime} \approx 0.2^{1}$.

Finally, there is a cooling distance associated with synchrotron radiation because of the magnetic field in the jet. This distance scale is

$$
L_{N}^{B}=\frac{6 \pi m_{p}^{4} c^{4}}{\sigma_{T} m_{e}^{2} E_{p}^{\prime} B^{\prime 2}} .
$$

Again, meson interaction lengths can be obtained by scaling by $\left(m_{M} / m_{p}\right)^{4}$.

The cooling times and the acceleration time for protons are shown in Fig. [1] For the proton flux, until cooling becomes important, acceleration dominates. For both the SJS and GRB example models, cooling through protonphoton interactions eventually dominates the acceleration. The crossover between acceleration time and $t_{p \gamma}$ in Fig. 1 occurs in an energy regime where $t_{p \gamma}$ is nearly

\footnotetext{
1 The Bethe-Heitler process $p \gamma \rightarrow p e^{+} e^{-}$is less effective than inelastic $p \gamma$ above the threshold [9] and we will therefore neglect it.
} 
constant, although there is strong energy dependence at lower energies.

When cooling dominates acceleration, the proton flux is cut off. Details of the cutoff depend on the energy dependence and mean inelasticity of the interaction [21, 25]. Generically, we can write

$$
\phi_{N}(E) \equiv \phi_{N}^{0}(E) f_{N}(E)=A E^{-2} f_{N}(E) .
$$

We apply a smooth cutoff $f_{N}(E)$ of the form of Eq. (20) in Ref. [21] with a spectral index $\Gamma=2$ and the parameter $\delta=1$ :

$$
f_{N}(E)=\left(1+\left(\frac{E}{E_{\max }}\right)^{\delta}\right) \exp \left[-\frac{(\Gamma-1)}{\delta}\left(\frac{E}{E_{\max }}\right)^{\delta}\right] .
$$

The energy at which the cross-over of acceleration and cooling times occurs, in the co-moving frame, is $E_{\max } \simeq$ $5.2 \times 10^{5} \mathrm{GeV}$ for the SJS and $E_{\max } \simeq 3.6 \times 10^{6} \mathrm{GeV}$ for the GRB model. Our result for the SJS model is in agreement with previous estimates of proton cooling times [9, 10].

Fig. 2 shows the meson cooling times and the decay times in the frame co-moving with the jet for both the SJS and GRB models. For mesons, the decay length and decay time includes the gamma factor (in the comoving frame), e.g., $L_{M}^{\mathrm{dec}}=\left(E_{M}^{\prime} / m_{M} c^{2}\right) c \tau_{M}$. The proper decay lengths $c \tau_{M}$ are listed in Table II Pion and kaon cooling times are in agreement with previous estimates [9, 10]. One can see from Fig. 2 that for $D$ mesons, the cross over of the decay time with the cooling time (hadronic or radiative) occurs at much higher energies than for pions and kaons.

\section{MESON AND NEUTRINO FLUXES}

Neutrinos are produced through meson production followed by meson decay. It is convenient to define the combined cooling length $\tilde{L}_{M}$ for meson $M$ through

$$
\tilde{L}_{M}^{-1}=\left(L_{N}^{\mathrm{had}}\right)^{-1}+\frac{m_{p}^{4}}{m_{M}^{4}}\left[\left(L_{N}^{I C}\right)^{-1}+\left(L_{N}^{B}\right)^{-1}\right]
$$

including the mass rescaling factor. An effective length for a given meson which also accounts for the decay is $\left(L_{M}^{\text {eff }}\right)^{-1}=\left(\tilde{L}_{M}\right)^{-1}+\left(L_{M}^{\text {dec }}\right)^{-1}$. With this definition, the equation describing the propagation of mesons $M$ in the jet is

$$
\frac{d \phi_{M}}{d x^{\prime}}=-\frac{\phi_{M}}{L_{M}^{\mathrm{eff}}}+Z_{N M} \frac{\phi_{N}}{\ell_{N}^{\mathrm{had}}}+Z_{N M}^{\gamma} \frac{\phi_{N}}{\ell_{N}^{\gamma}},
$$

where $Z_{N M}$ are the $Z$-moments for $p p$ meson production and $Z_{N M}^{\gamma}$ are the $Z$-moments for $p \gamma$ meson production. We have here assumed a constant density that does not vary with $x^{\prime}$. Finally, the neutrino flux from meson $M$ is obtained from the meson flux by integrating the equation

$$
\frac{d \phi_{\nu}}{d x^{\prime}}=Z_{M \nu} \frac{\phi_{M}}{L_{M}^{\mathrm{dec}}}
$$

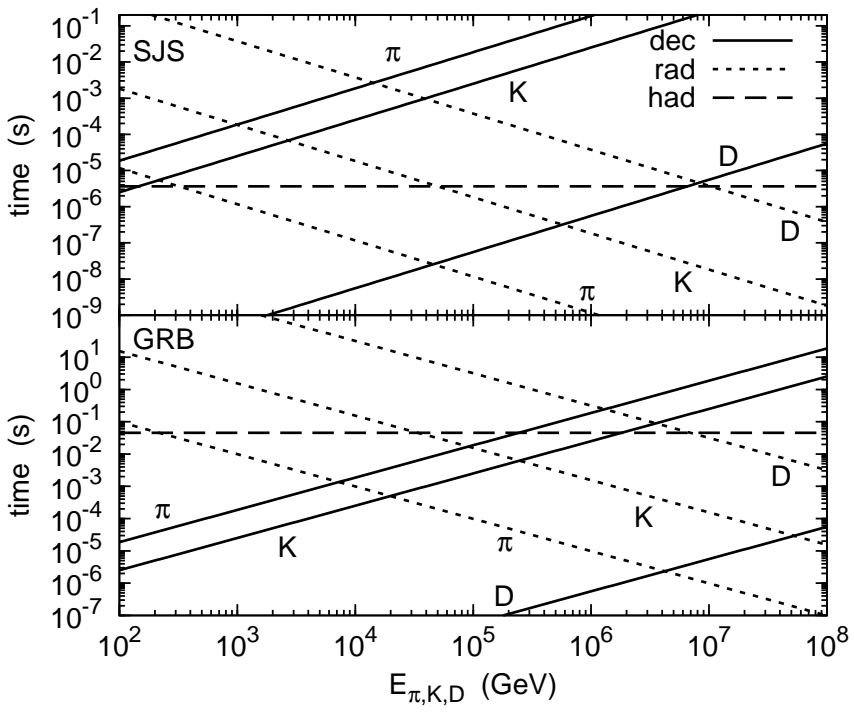

FIG. 2: Meson cooling times for SJS and GRB in the frame co-moving with the jet.

where $Z_{M \nu}$ is the decay $Z$-moment listed in Table II] In the limit $x^{\prime} \rightarrow \infty$ we have

$$
\phi_{\nu}\left(E^{\prime}\right)=Z_{M \nu} \frac{L_{M}^{\mathrm{eff}}}{L_{M}^{\mathrm{dec}}} \frac{Z_{N M} \ell_{N}^{\gamma}+Z_{N M}^{\gamma} \ell_{N}^{\mathrm{had}}}{\ell_{N}^{\mathrm{had}}+\ell_{N}^{\gamma}} \phi_{N}\left(E^{\prime}\right) .
$$

This has the correct limiting behavior, e.g., when decays dominate the meson effective length $L_{M}^{\mathrm{eff}}$ and the energy is below the $p \gamma$ cross section threshold. In this limit,

$$
\phi_{\nu}\left(E^{\prime}\right)=Z_{M \nu} Z_{N M} \phi_{N}\left(E^{\prime}\right),
$$

essentially the same as in Ref. [10] at low energy, however, this form has a smoother transition to the high energy behavior.

We calculate the $Z$-moments for charm production taking into account the effects of parton saturation at high energies as in Ref. [26]. These moments are energy dependent, with weak dependence at higher energies. In our calculation of the neutrino flux, for simplicity we use the constant value of the $Z$-moments for charm given in Table [I] We neglect the production of other charmed particles. We estimate that the inclusion of $\Lambda_{c}$ production would increase the flux of neutrinos from charm by $\sim 15 \%$ [27]. For $\pi^{ \pm}$and $K^{ \pm}$production, however, we assume Feynman scaling, leading to energy independent $Z$-moments. To compute the $Z$-moments for $p p$ we use the parametrization of the rapidity distribution [28]

$$
\frac{d N_{\pi}}{d x_{E}}=0.12 \frac{\left(1-x_{E}\right)^{2.6}}{x_{E}^{2}}
$$

where $x_{E}=E_{\pi} / E_{p}$, and for $p \gamma$ we fit this form to the data for $\pi^{0}$ production in Ref. [29]. For $K^{ \pm}$we take the $\pi^{ \pm}$result rescaled by 0.1 [30]. The decay $Z$-moments are 


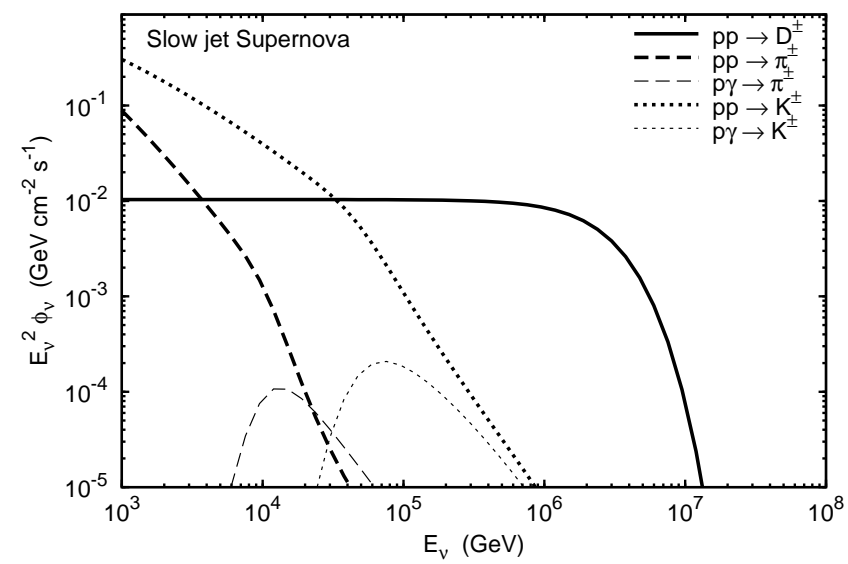

FIG. 3: Muon neutrino plus antineutrino flux from a slow jet supernova in the observer's frame, without including neutrino oscillation effects as they propagate on their way to Earth.

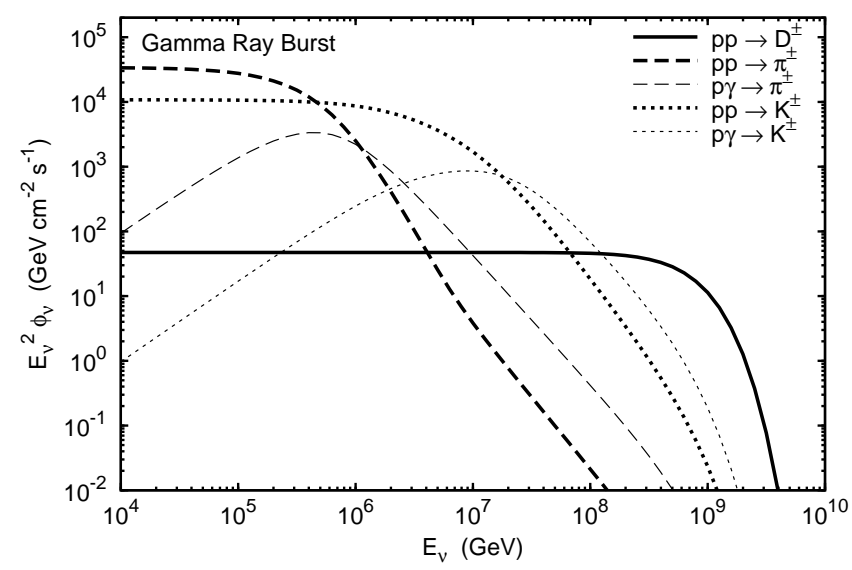

FIG. 4: Muon neutrino plus antineutrino flux from a gamma ray burst in the observer's frame, without including neutrino oscillation effects as they propagate on their way to Earth.

calculated using the expressions in Refs. [31, 32]. The values for $Z$-moments are shown in Table

To evaluate the neutrino flux from a source in the Earth's frame, co-moving energies are scaled by the jet bulk Lorentz factor $\Gamma_{j}$. The neutrino flux is related to the jet luminosity $L_{j}$, the distance to the source $d_{L}$, taken here to be $20 \mathrm{Mpc}=6.17 \times 10^{25} \mathrm{~cm}$, and the jet opening angle shown in Table I. The neutrino flux is

$$
\begin{aligned}
\phi_{\nu}(E) & =Z_{M \nu} \frac{L_{M}^{\mathrm{eff}}}{L_{M}^{\mathrm{dec}}\left(\ell_{N}^{\mathrm{had}}+\ell_{N}^{\gamma}\right)} \\
& \times\left(Z_{N M} \ell_{N}^{\gamma}+Z_{N M}^{\gamma} \ell_{N}^{\text {had }}\right) \\
& \times \frac{L_{j} \Gamma_{j}^{2}}{2 \pi \theta_{j}^{2} d_{L}^{2} \ln \left(E_{\max }^{\prime} / E_{\min }^{\prime}\right)} E^{-2} .
\end{aligned}
$$

The quantity $E_{\max }^{\prime}$ is the energy where cooling and ac-
TABLE II: $Z$-moments and decay lengths used in the evaluation of the muon neutrino plus antineutrino flux. The $p \rightarrow p$ moment is taken to be $Z_{N N}^{\text {had }}=0.5$.

\begin{tabular}{ccccc}
\hline$M$ & $Z_{M \nu}$ & $Z_{N M}$ & $Z_{N M}^{\gamma}$ & $c \tau[\mathrm{cm}]$ \\
\hline$\pi^{ \pm}$ & 0.061 & 0.55 & 0.13 & 780 \\
$K^{ \pm}$ & 0.19 & 0.055 & 0.0065 & 370 \\
$D^{ \pm}$ & 0.045 & $2.4 \times 10^{-3}$ & - & $3.2 \times 10^{-2}$ \\
$D^{0}$ & 0.017 & $5.6 \times 10^{-3}$ & - & $1.2 \times 10^{-2}$ \\
\hline
\end{tabular}

celeration cross over, and $E_{\min }^{\prime}=m_{p} c^{2}$ is the proton's rest energy. In Fig. 3 we show the neutrino flux, $\phi_{\nu}$, as a function of neutrino energy for the slow jet supernova model, and in Fig. 4, for the gamma ray burst model. The fluxes shown are from $p p$ and $p \gamma$ interactions. At low energies, the dominant sources of neutrinos are pions and kaons produced in $p p$ interactions. Above approximately $E \simeq 10^{7} \mathrm{GeV}, p \gamma$ production of pions and kaons becomes comparable in strength for the GRB, while for the SJS it is subdominant for all energies.

At low energies, where cooling is not important for the $D$ mesons, the $D^{+}$and $D^{0}$ contributions are approximately equal using the parameters in Table II At high energies, $p p$ production of charmed mesons, with their shorter lifetimes, dominate although the maximum proton energy in the GRB jet restricts the dominance of the neutrino flux from charm to a narrow energy interval. As noted in Eq. (5), the acceleration time depends on unknowns including $\kappa$. If $\kappa=1$, the maximum proton energy in the comoving frame increases by a factor of 10 , increasing the range of energies for which charm decays contribute to the neutrino flux.

To discuss the potential for neutrino flux attenuation in the source, we review the proton and neutrino optical depths for the slow jet supernova model, where the jet does not emerge from the source. The jet propagates to $r_{j} \sim 5 \times 10^{10} \mathrm{~cm}$ in the SJS model. The optical depth $\tau^{\prime}=\sigma n^{\prime} r_{j} / \Gamma_{b}$ for protons in $p p$ and $p \gamma$ interactions are

$$
\begin{aligned}
\tau_{p p}^{\prime} & \simeq 3 \times 10^{5} \\
\tau_{p \gamma}^{\prime} & \simeq 10^{6} \text { above threshold }
\end{aligned}
$$

The proton-proton cross section is a factor of $10^{8}$ larger than the neutrino cross section with protons at $E_{\nu}=10^{6}$ $\mathrm{GeV}$. At high energies, the cross section scales like $E_{\nu}^{0.44}$, so even at $E_{\nu}=10^{8} \mathrm{GeV}$, the proton cross section is a factor of more than $10^{7}$ larger than the neutrino cross section. At $10^{6} \mathrm{GeV}$, the optical depth for the neutrino within the jet is

$$
\tau_{\nu p}^{\prime} \simeq 2 \times 10^{-2} .
$$

Neutrino attenuation within the jet is therefore not important.

The densities inside and outside of the jet are not the same. In the SJS model, the jet does not reach the stellar envelope, however, $r_{j}$ is large enough that the relevant densities would be for He or H. Taking as an example 33. 
$\rho=\left(r_{j} / r\right)^{3} \mathrm{~g} / \mathrm{cm}^{3}$ for the He density, the optical depth for a $10^{6} \mathrm{GeV}$ neutrino is $\tau_{\nu p} \simeq 10$. On the other hand, if the relevant density is for the $\mathrm{H}$ envelope, the density is much lower and the optical depth $\tau_{\nu p} \ll 1$. This would indicate that some attenuation may be important in a full evaluation of neutrino fluxes for these types of sources with a more detailed model of the stellar exterior [14]. For GRBs, the hadronic jet emerges, so given that the neutrino-nucleon cross section is so much lower than the proton-proton cross section, neutrino attenuation in GRBs should not be important.

Taking into account the uncertainty in the acceleration differential index does not have a significant effect on the relative importance of the various contributions to the neutrino flux. The high energy neutrino flux in the two examples shown here is dominated by charmed meson decay. In contrast to pions and kaons, charmed mesons give equal amounts of $\nu_{e}+\bar{\nu}_{e}$ and $\nu_{\mu}+\bar{\nu}_{\mu}$ at the source since the neutrinos are coming from semileptonic decays. This could give different flavor ratios of neutrinos in neutrino telescopes at very high energies. This has also been noted in Ref. [8]. Since many properties of astrophysical sources are not well understood, neutrino measurements may hold the key to understanding the environments in which they were produced. Charm production in astrophysical jets provides an important enhancement of the neutrino flux at very high energies.

Current neutrino experiments, such as IceCube which has only partial implementation of the strings, have already put some limits on neutrino flux from a specific astrophysical source [34]. Once all the strings are implemented in IceCube, the sensitivity for detection of the astrophysical neutrinos will improve significantly. In addition, future cubic kilometer deep-sea neutrino detector [35] will also have a very good chance to look for astrophysical neutrinos from point sources.

\section{Acknowledgments}

This research was supported by US Department of Energy contracts DE-FG02-91ER40664, DE-FG0204ER41319 and DE-FG02-04ER41298.
[1] J. Ahrens et al., Astropart. Phys. 20, 507 (2004).

[2] U. F. Katz, Nucl. Instrum. Meth. A 567, 457 (2006).

[3] I. Kravchenko et al., Phys. Rev. D 73, 082002 (2006); Int. J. Mod. Phys. A 21S1, 153 (2006).

[4] See e.g., J. Vandenbroucke, G. Gratta and N. Lehtinen, Astrophys. J. 621, 301 (2005).

[5] S. E. Woosley, Astrophys. J. 405, 273 (1993).

[6] P. Mészáros, Rept. Prog. Phys. 69, 2259 (2006).

[7] E. Waxman and J. N. Bahcall, Phys. Rev. Lett. 78, 2292 (1997).

[8] M. Kachelriess and R. Tomas, Phys. Rev. D 74, 063009 (2006).

[9] S. Razzaque, P. Mészáros and E. Waxman, Phys. Rev. Lett. 93, 181101 (2004) [Erratum-ibid. 94, 109903 (2005)]; Mod. Phys. Lett. A 20, 2351 (2005).

[10] S. Ando and J. F. Beacom, Phys. Rev. Lett. 95, 061103 (2005).

[11] See, however, H. B. J. Koers and R. A. M. Wijers, arXiv:0711.4791.

[12] P. Mészáros and E. Waxman, Phys. Rev. Lett. 87, 171102 (2001).

[13] S. Razzaque et al., Phys. Rev. D 68, 083001 (2003).

[14] S. Horiuchi and S. Ando, Phys. Rev. D 77, 063007 (2008).

[15] X. Y. M. Wang and Z. G. M. Dai, arXiv:0807.0290

[16] K. Murase, Phys. Rev. D 78, 101302 (2008).

[17] M. Kachelriess, S. Ostapchenko and R. Tomas, Phys. Rev. D 77, 023007 (2008).

[18] D. Band et al., Astrophys. J. 413, 281 (1993).

[19] R. D. Preece, M. S. Briggs, R. S. Mallozzi, G. N. Pendleton, W. S. Paciesas and D. L. Band, Astrophys. J. Suppl.
126, 19 (2000).

[20] J. Giacalone and J. R. Jokipii, J. Phys. Conf. Ser. 47, 160 (2006).

[21] R. J. Protheroe and T. Stanev, Astropart. Phys. 10, 185 (1999).

[22] T. K. Gaisser, Cosmic rays and particle physics, Cambridge University Press (1990).

[23] M. Thunman et al., G. Ingelman and P. Gondolo, Astropart. Phys. 5, 309 (1996).

[24] A. Mucke, R. Engel, J. P. Rachen, R. J. Protheroe and T. Stanev, Comput. Phys. Commun. 124, 290 (2000)

[25] R. J. Protheroe, Astropart. Phys. 21, 415 (2004).

[26] R. Enberg, M. H. Reno and I. Sarcevic, Phys. Rev. D 78, 043005 (2008).

[27] See, e.g., L. Pasquali, M. H. Reno and I. Sarcevic, Phys. Rev. D 59, 034020 (1999) arXiv:hep-ph/9806428.

[28] C. G. S. Costa, F. Halzen and C. Salles, Phys. Rev. D 52, 3890 (1995).

[29] C. Adloff et al., Eur. Phys. J. C 18, 293 (2000).

[30] G. J. Alner et al., Phys. Rept. 154, 247 (1987).

[31] P. Lipari, Astropart. Phys. 1, 195 (1993).

[32] E. V. Bugaev, S. Sinegovskaya, S. I. Sinegovsky and N. Takahashi, Phys. Rev. D 58, 054001 (1998).

[33] P. Meszaros and M. J. Rees, Astrophys. J. 556, L37 (2001).

[34] R. Abbasi et al. [IceCube Collaboration], arXiv:0902.0131 [astro-ph.HE].

[35] D. Dornic and G. Lelaizant, arXiv:0810.1452 [astro-ph]. 